\title{
Localization of Proliferating Cell Nuclear Antigen in Aural Cholesteatoma
}

\author{
NAOMI UCHIDA, SHINSUKE ITO AND MINORU HIRANO \\ Department of Otolaryngology, Kurume University School of Medicine, \\ Kurume, 830 Japan
}

Received for publication October 13, 1993

\begin{abstract}
Summary: Middle ear cholesteatoma is not a genuine tumor but has a remarkable proliferative activity which causes serious destruction of the mastoid bone. In the present study, we used immunohistochemistry with antiproliferating cell nuclear antigen (PCNA) antibody on cholesteatomatous tissues to evaluate the localization of PCNA, as it has been said that PCNA is a very available protein for showing cell proliferative activity. Moderately concentrated PCNA was demonstrated within the germinal basal layer cells of the cholesteatomatous epithelium in three of eight surgical specimens. Furthermore, in one case of very active osteolytic cholesteatoma, PCNA activity was demonstrated in the mesenchymal cells, probably fibroblast-like cells, in direct contact to the destroying mastoid bone lesions. Although the etiology and histopathology of the invasive and proliferative activity of middle ear cholesteatomatous tissues are unclear, this observation suggests that immunohistopathological examination using PCNA antibody might be a useful tool for evaluating bone resorption activity and for establishing the prognosis of various types of cholesteatoma.
\end{abstract}

Key words: cholesteatoma - proliferating cell nuclear antigen - immunohistopathology - epithelium - granulation

\section{Introduction}

Recently cellular proliferation has been studied by immunohistochemical staining method for proliferating cell nuclear antigen (PCNA), also called cyclin, which appears in the nucleus during the synthetic phase of the cell cycle (Oda et al. 1993; Schiffer et al. 1993, Tateyama et al. 1993). In this report, we investigated the localization of PCNA in middle ear cholesteatomatous epithelium and granulation of eight surgical cases. Cholesteatoma is not a genuine "tumor" but shows very frequently remarkable proliferative activity and causes mastoid bone destruction and resorption (Harris, 1962; Moriyama et al. 1987).

\section{Materials and Methods}

The tissues used in this study were obtained during surgical procedures (tympanoplasty) of the middle and inner ear in the Department of Otolaryngology, Kurume University Hospital, Kurume, 1993. Specimens were obtained from eight patients (Table 1) with cholesteatoma and were fixed in $10 \%$ formaldehyde solution. Of those cases, three cases (case 1-3) had an aggressive type-cholesteatoma showing remarkable bone resorption in the mastoid lesions. About three to five blocks of tissue were obtained in each case. Histological diagnosis of all the specimens was made with routine hematoxylin and eosin staining. Normal 
tissue used for positive and negative controls included human skin, tonsil and external aural canal. Immunohistochemistry was utilized to examine PCNA. The antibody was purchased from DAKOPATTS, Copenhagen, Denmark. The conventional $4-\mu \mathrm{m}$ sections were dewaxed, soaked in alcohol, and immersed for $30 \mathrm{~min}$ in $25 \%$ phosphatebuffered saline in methanol, with $0.5 \%$ hydrogen peroxide to block endogenous peroxidase activity. The sections were incubated with PCNA monoclonal antibody, PC10 diluted at $1: 200$. A subsequent reaction was made by using some parts of a PAP kit (DAKO). The sections were stained with freshly prepared diaminobenzidine solution and partly counterstained with hematoxylin. Tissues from a normal human tonsil was used as a positive control. Substitution of the primary antibody by phosphate-buffered saline was used as a negative control. Some tissues were decalcinated in advance with the Plank - Rychlo method.

\section{Results}

The results of the immunohistological studies are summarized in Table 1. Positive PCNA reactivity was demonstrated in the specimens of four patients (case
1-4). In the case 1,2 and 4 , PCNA was expressed intensely within the basal layer cells in the cholesteatomatous epithelium (Fig. 1). The keratinized squamous epithelium layer of these cases was relatively thin in contrast to the epithelium of the other cases which were negative for PCNA (Fig. 2). Furthermore, in the case 3 , just at the edge of the areas of bone destruction, some of mesenchymal cells, including fibroblasts, showed moderately positive reactivity for PCNA in the granulomatous tissues (Fig. 3). In keratinized epithelial debris, superficial cell layers, Langerhans cells and other inflammatory cells, capillary endothelial cells in the granuloma and matrix were negative for PCNA.

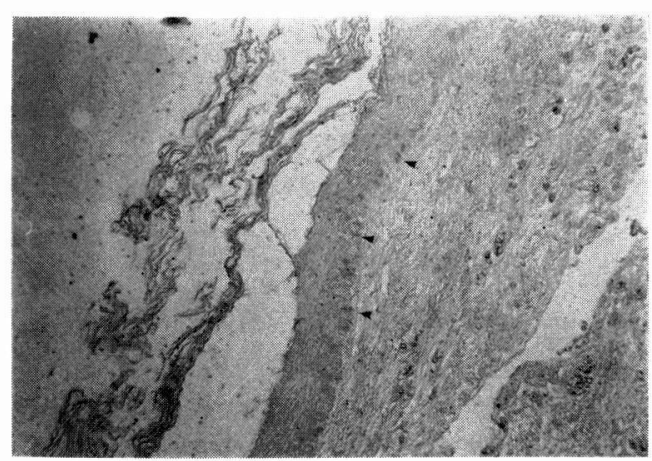

Fig. 1. PCNA was expressed within the basal layer cells (V) [Immunohistochemistry for PCNA, $\times 100]$

TABLE 1.

Summary of the clinical and histological findings in the eight cholesteatoma cases

\begin{tabular}{c|c|c|c|c|c}
\hline Case & Age/Sex & Otorrhea & bone destruction & epithelium & PCNA \\
\hline 1 & $42 / \mathrm{M}$ & + & $\mathrm{H}$ & thin & ++ \\
2 & $11 / \mathrm{M}$ & + & + & thin & ++ \\
3 & $58 / \mathrm{F}$ & - & $\mathrm{H}$ & thin & ++ \\
4 & $48 / \mathrm{F}$ & + & $\mathrm{H}$ & thin & ++ \\
5 & $62 / \mathrm{M}$ & - & + & thin & -- \\
6 & $8 / \mathrm{F}$ & + & - & thick & -- \\
7 & $18 / \mathrm{F}$ & + & - & thick & - \\
8 & $55 / \mathrm{F}$ & - & - & thick & - \\
\hline
\end{tabular}




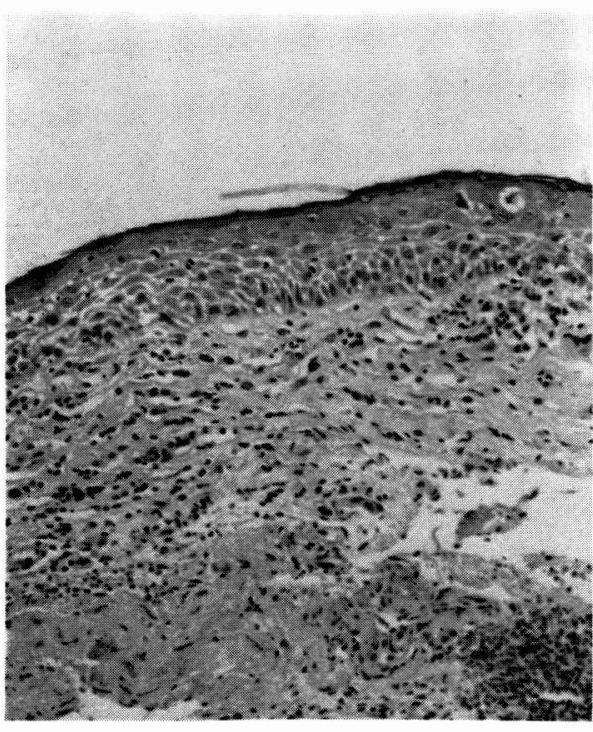

left

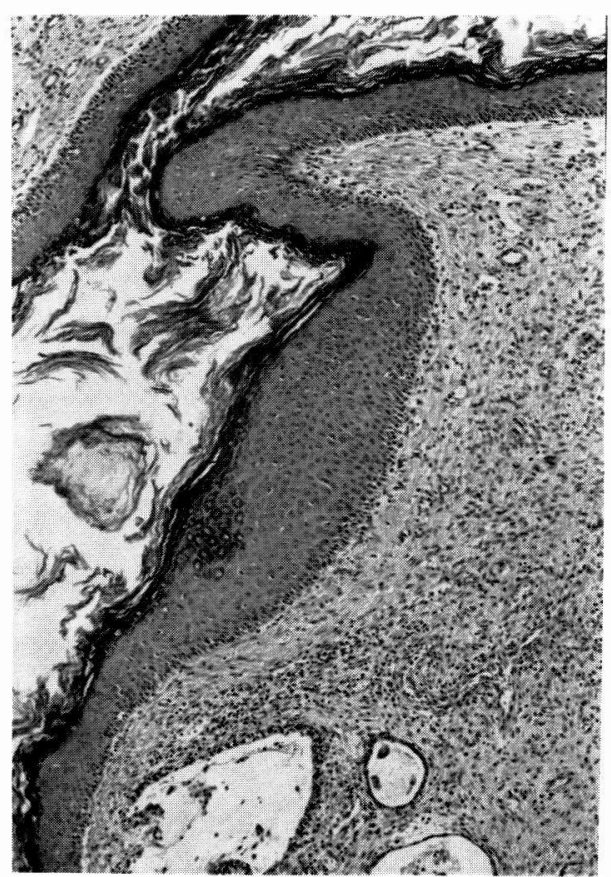

right

Fig. 2. Epithelium layer was thin [left; positive case, right; negative case-7, H.E. $\times 200$ and $\times 100]$ in case 1 .

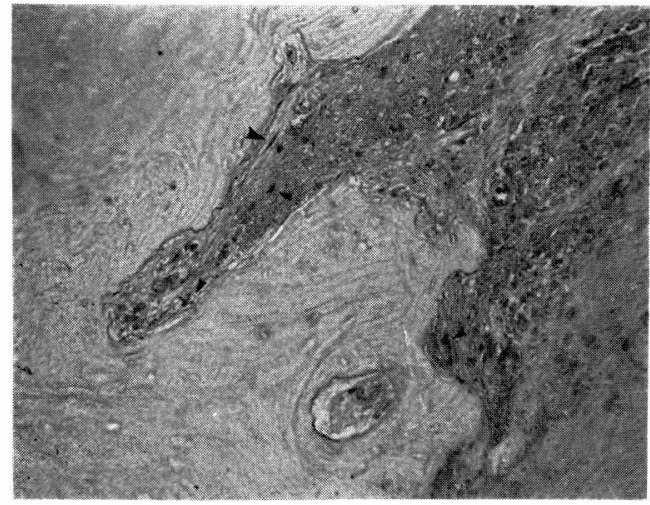

Fig. 3. Some of the mesenchymal cells, probably fibroblast-like cells, showed positive reactivity for PCNA (V) [Immunohistopathology for PCNA, $\times 100]$

\section{Discussion}

Recently, various new methods and antigens have become available for the measurement of cellular proliferation. One such protein, PCNA, also called cyclin, is a $36 \mathrm{kd}$ proliferation-associated, intranuclear polypeptide antigen (Morris and Mathews, 1989; Mori et al. 1993). Expression of this antigen is closely linked to the cell cycle. Furthermore, this protein can be detected in formalinfixed, paraffin-embedded sections of surgical samples (Garcia et al. 1989). So, in this report, we tried to detect PCNA in cholesteatomatous tissues of surgical specimens, because middle ear cholesteatoma frequently shows remarkable proliferative activity to invade and erode the surrounding bone tissue. The resulting bone resorption and destruction cause 
sometimes serious complications, such as meningitis and sepsis. Here, immunohistopathological examination revealed that the germinal basal layer cells of very active type-cholesteatoma's epithelium and fibroblast-like cells in the matrix just adjacent to the bone destruction lesions showed definitely positive reactivity for PCNA. The etiology and physiopathology of the osteolysis and of the abnormal proliferative property of the cholesteatomatous epithelial cells and subepithelial granulomatous tissues are unclear. We would like to examine more specimens of various types of so-called active cholesteatoma, in particular, of children's serious aggressive type, in order to study the mechanism of the rapid osteolysis in the mastoid tissue. Furthermore, in other cases, immunostaining of PCNA may be also one of the criteria suggesting the prognosis of the cholesteatoma.

\section{References}

Garcia, R. L., Coltrera, M.D. and Gown, A.M. (1989). Analysis of proliferative grade using anti-PCNA/Cyclin monoclonal antibodies in fixed, embedded tissues. Comparison with flow cytometric analysis. Am. J. Pathol. 134, 733-739.
HARRIS, A.J. (1962). Cholesteatosis and chronic otitis media. The histopathology of osseous and soft tissues. Laryngoscope 72, 954-980.

Mori, M., KaKejI, Y., Adachi, Y., Moriguchi, S., Maehara, Y. et al. (1993). The prognostic significance of proliferating cell nuclear antigen in clinical gastric cancer. Surgery 113, 683-690.

Moriyama, H., Honda, Y., Huang, C. C. and Abramson, M. (1987). Bone resorption in cholesteatoma: epithelial-mesenchymal cell interaction and collagenase production. Laryngoscope $97,854-859$.

Morris, G. F. and Mathews, M. B. (1989). Regulation of proliferating cell nuclear antigen during the cell cycle. J. Biol. Chem. 264, 13856-13864.

Oda, Y., Hashimoto, H., Takeshita, S. and Tsuneyoshi, M. (1993). The prognostic value of immunohistochemical staining for prolif erating cell nuclear antigen in synovial sarcoma. Cancer 72, 478-485.

Schiffer, D., Chio, A., Giordana, M. T., Pezzulo, T. and Vigliani, M.C. (1993). Proliferating cell nuclear antigen expression in brain tumors, and its prognostic role in ependymomas: an immunohistochemical study. Acta Neuropathol. 85, 495-502.

Tateyama, H., Mizuno, T., Tada, T., Eimoto, T. Hashimoto, T. et al. (1993). Thymic epithelial tumours: evaluation of malignant grade by quantification of proliferating cell nuclear antigen and nucleolar organizer regions. Virchows Archiv. Pathol. Anat.422, 265-269. 